\title{
Diagnostic Accuracy of Two Molecular Tools for Diagnosis of Congenital Chagas Disease
}

\author{
Margarita María Catalina Bisio, ${ }^{1,2}$ - Rocío Rivero ${ }^{3}$. Nicolás Gonzalez ${ }^{1}$ Griselda Ballering ${ }^{1} \cdot$ Indira D’Amico $^{1}$. \\ Camila Kessler ${ }^{2}$. Samanta Moroni ${ }^{1}$ - Guillermo Moscatelli ${ }^{1,2}$. Andrés Mariano Ruiz ${ }^{3,4}$. Jaime Altcheh ${ }^{1,2}$
}

Accepted: 27 July 2021 / Published online: 23 August 2021

(c) The Author(s), under exclusive licence to Springer Nature Switzerland AG 2021

\begin{abstract}
Background and Objective The real prevalence of congenital Chagas disease is undefined because of difficulties in the detection of Trypanosoma cruzi by microscopic examination. The aim of this study was to determine the diagnostic accuracy of two molecular diagnostic tools, qPCR and LAMP, in the diagnosis of congenital Chagas disease in a clinical setting.

Methods To this end, we conducted a prospective cohort study in a tertiary care center, of infants under 9 months of age, born in Buenos Aires to women with Chagas disease. Blood samples were collected for microscopic examination and molecular diagnosis at baseline. If negative, infants were followed up until 9 months of age to determine a final diagnosis by serology. In-house qPCR and LAMP previously validated were challenged as index tests.

Results A total of 154 participants were potentially eligible, 120 of whom were enrolled. Finally, 102 (66.2\%) of them fulfilled the follow-up. The diagnosis of congenital Chagas disease was confirmed in 13 infants and excluded in 89. Both the sensitivity and specificity of the qPCR were $100.0 \%$ (95\% confidence interval $75.3-100.0$ and $95 \%$ confidence interval 95.9-100.0, respectively), whereas the sensitivity and specificity of LAMP were 69.2\% (95\% confidence interval 38.6-90.9) and $100 \%$ (95\% confidence interval 95.9-100.0), respectively.

Conclusions The qPCR agreed with the current diagnostic algorithm, and was a reliable and sensitive tool to detect congenital Chagas disease earlier, providing an appropriate and timely identification of infected infants requiring treatment. LAMP was able to detect congenital Chagas disease in infected infants by naked-eye visualization in accordance with a microscopic examination. The advantages of molecular diagnostic tools should be taken into account by the health system to improve congenital Chagas disease diagnosis.
\end{abstract}

\section{Introduction}

Margarita M. C. Bisio and Rocío Rivero contributed equally to this work. Author order was determined on the basis of seniority.

Margarita María Catalina Bisio

marguib@gmail.com

$\bowtie$ Jaime Altcheh jaltcheh@gmail.com

1 Servicio de Parasitología y Enfermedad de Chagas, Hospital de Niños “Dr. Ricardo Gutiérrez”, CP 1425 Gallo 1330, Buenos Aires, Argentina

2 Instituto Multidisciplinario de Investigaciones en Patologías Pediátricas, Consejo Nacional de Investigaciones Científicas y Técnicas (CONICET-GCBA), Buenos Aires, Argentina

3 Instituto Nacional de Parasitología Dr. Mario Fatala Chaben, Buenos Aires, Argentina

4 Consejo Nacional de Investigaciones Científicas y Técnicas (CONICET), Buenos Aires, Argentina
Trypanosoma cruzi is the etiological agent of Chagas disease. In this zoonotic disease, the parasite is transmitted by different routes, among which congenital transmission is the most important both in endemic areas where vector populations are under control and in non-endemic areas [1]. In endemic countries, it is estimated that between 8000 and 15,000 T. cruzi-infected infants are born to infected mothers every year. Early diagnosis of congenital Chagas disease (CCD) is considered a cost-effective strategy as, in contrast to the disease in adults, the cure rate in infants under 1 year of age is almost $100 \%$ and tolerance to treatment is acceptable [2,3]. However, current diagnosis of CCD in infants is complex and requires an algorithm based on various tests performed during an 8- to 10-month follow-up [1]. Parasitological diagnosis in infants involves concentration techniques via centrifugation using capillary or microtubes for 


\section{Key Points}

Current diagnosis of congenital Chagas disease is complex and requires an algorithm based on various tests performed during an 8- to 10-month follow-up. A late diagnosis results in missed opportunities for the treatment of infected infants.

Although several studies have shown the high sensitivity of PCR in congenital Chagas disease diagnosis, in most Latin American countries, this tool had not been validated in routine screening and its implementation is still limited. There is a need to obtain more evidence in order to modify current practice.

Our study evidenced moderate and high overall accuracy of LAMP and qPCR, respectively. Considering the advantages and limitations of these molecular diagnostic tools, these should be taken into account by the health system to improve early diagnosis and treatment of congenital Chagas disease.

the detection of parasites from the buffy coat by microscopic examination (ME) $[1,4,5]$. Although these techniques concentrate parasites, they have limited clinical sensitivity because of multiple factors, including the parasitic loads and technical issues such as operator dependence [6]. Additionally, no quality-control system may be available for ME [7]. Therefore, CCD diagnosis in infants relies on serological methods at 8-10 months, once maternal antibodies become undetectable [1].

In endemic countries where the primary healthcare system is weak and the vulnerable population does not have access to the healthcare system, the above-mentioned diagnostic algorithm has been difficult to implement. In Argentina, the available data show deficiencies in the follow-up of infants and CCD notification, and the real incidence and clinical burden of this disease are unknown [8]. In fact, the Pan American Health Organization recognizes that CCD may be underestimated because maternal and infant healthcare centers do not routinely screen mothers and/or infants, and thus recommends actively exploring opportunities to diagnose and cure $\geq 90 \%$ of infants with CCD [9].

In this context, an alternative or complement to the current CCD diagnostic algorithm could be parasite DNA detection using molecular diagnostic tools, but their implementation in endemic regions remains limited to reference centers [10]. Over 20 years, the PCR technique has been presented as a promising tool for sensitive and specific detection of $T$. cruzi parasites [11-13]. Furthermore, isothermal DNA amplification (LAMP), which requires less infrastructure, has been reported as a possible molecular diagnostic tool for T. cruzi detection [14-16]. Before implementation into routine clinical practice and incorporation into guidelines, every new diagnostic assay should go through a lengthy validation process. Although both T. cruzi qPCR and LAMP have been analytically validated [14, 17], they have not yet been clinically validated to assess their clinical utility. Thus, the aim of this study was to determine the diagnostic accuracy of qPCR and LAMP previously analytically validated in the diagnosis of CCD in a clinical setting, using the current diagnostic algorithm as the diagnostic reference standard. In vitro diagnostics assays are not commercially available for qPCR or LAMP. Therefore, two in-house assays whose validations had been published and could be performed in the clinical setting were selected: a PCR assay targeting the T. cruzi nuclear satellite and a LAMP assay targeting $18 \mathrm{~S}$ rRNA genes.

\section{Materials and Methods}

\subsection{Study Population}

The samples analyzed were obtained in the context of a prospective cohort study conducted in infants. A total of 154 potentially eligible infants born to $T$. cruzi-infected mothers who attended the Parasitological and Chagas Service of the Hospital de Niños "Ricardo Gutiérrez", Buenos Aires, Argentina, for a CCD diagnosis between July 2012 and December 2015 were consecutively recruited for this study following Good Clinical Practices. The accuracy of both qPCR and LAMP was evaluated according to the Standards for the Reporting of Diagnostic Accuracy Studies (STARD) statement [18].

Subjects meeting the following criteria were considered for inclusion: infants aged under 9 months, $T$. cruzi maternal serology reactive by two serological tests, and at least one blood sample available for molecular diagnosis. Exclusion criteria were: mother or infants who had received previous treatment for Chagas disease; infants born in or who had traveled to an area endemic for Chagas disease.

\subsection{Reference Standard by the Current CCD Diagnostic Algorithm}

All infants recruited were screened for $T$. cruzi infection according to the official guidelines [19]. Briefly, infection in infants under 9 months of age was assessed by ME performed by a trained biochemist with 10 years of experience using the microhematocrit method [20]. In the case of a negative $\mathrm{ME}$ result, infants were followed up every 3 months until 9 months of age by ME. Infection of T. cruzi in infants aged older than 9 months was confirmed or discarded by 
two serological tests currently used in Argentina: Chagatest ELISA recombinante v.3.0 (Wiener Lab, Rosario, Argentina) and indirect hemagglutination Chagatest-HAI (Wiener Lab) (Fig. 1). Because at least two positive serological tests are necessary to consider a patient to be infected, as recommended by the World Health Organization and the Pan American Health Organization [21], in cases of inconclusive serological results, a passive particle agglutination test (Bayer, Buenos Aires, Argentina) was performed. All three assays are validated commercial tests widely used in clinical settings. In short, infants were considered infected when an ME before 9 months or two serological tests over 9 months were positive, whereas they were considered not infected when both ME and two serological tests over 9 months were negative. This algorithm was the reference standard.

\subsection{Sample Collection for Index Tests}

Venous blood samples for molecular diagnosis were obtained by direct puncture of the antecubital area of the arm at baseline (T1), when infants were recruited, and at the end of the follow-up (T2), at 9 months of age (Fig. 1). Samples were preserved as previously described [12]. Depending on the likelihood of being able to obtain a certain blood volume in each procedure, we chose to collect $0.5,1$, or $2 \mathrm{~mL}$ of blood, which was preserved in a tube containing the same volume of guanidine 6M-EDTA 0.2 M buffer (GE buffer) and, after $48-72 \mathrm{~h}$, samples were heated at $100{ }^{\circ} \mathrm{C}$ for 5,10 , or $15 \mathrm{~min}$ respectively and stored at $4{ }^{\circ} \mathrm{C}$ until processing. Samples at $\mathrm{T} 1$ were then analyzed by the two index tests chosen: qPCR and LAMP to assess the diagnostic accuracy. Samples at T2 were then analyzed by qPCR to establish the parasitic load in those cases with positive results. Laboratory technicians ascertaining the results of the qPCR and LAMP were blinded to the results of the reference standard.

\subsection{DNA Extraction}

Samples were processed using the High Pure PCR Template Preparation Kit (Roche Diagnostics Corp., Indianapolis, IN, USA), according to the manufacturer's instructions with slight modifications. Namely, $5 \mu \mathrm{L}$ of internal positive control (IPC, previously described by Duffy et al. [17]) was added to $300 \mu \mathrm{L}$ of each sample and this mix was processed [22]. Each round of DNA extraction was performed using ten blood samples: eight from patients, one from a negative control for $T$. cruzi infection, and one from a positive extraction control (see below). After extraction, DNA was stored at $-20{ }^{\circ} \mathrm{C}$ until use in the qPCR or LAMP assays.

\subsection{PCR Reaction}

A multiplex qPCR assay able to quantify the T. cruzi nuclear satellite and IPC DNA in a single-tube reaction previously analytically validated was implemented [22]. The qPCR reactions were carried out with $5 \mu \mathrm{L}$ of eluted DNA, using FastStart Universal Probe Master Mix (Roche Diagnostics GmbHCorp, Mannheim, Germany) in a final volume of 20 $\mu \mathrm{L}$. The amplifications were carried out using $750 \mathrm{nM}$ of the cruzi1 and cruzi 2 primers, $50 \mathrm{nM}$ of the cruzi3 probe, $100 \mathrm{nM}$ of the IACTqFw and IACTqRv primers, and $50 \mathrm{nM}$ of the IACTq probe. The TaqMan MGB cruzi3 and IACTq probes were labeled with FAM and VIC dye, respectively. The cycling conditions were as follows: $10 \mathrm{~min}$ at $95^{\circ} \mathrm{C}$, and 40 cycles of $15 \mathrm{~s}$ at $95^{\circ} \mathrm{C}$, followed by $1 \mathrm{~min}$ at $58^{\circ} \mathrm{C}$. The amplifications were carried out in a StepOne Real Time PCR System (Applied Biosystems, Foster City, CA, USA). Two negative controls (water and DNA from non-infected human samples) and two positive amplification controls (5 and $50 \mathrm{fg}$ of DNA purified from T. cruzi trypomastigotes per tube) were included in every round. To analyze the results, the threshold was set at 0.02 and 0.01 for $T$. cruzi and IPC, respectively. Samples that yielded negative IPC results were discarded. Clinical samples were considered positive when the fluorescent signals crossed the threshold.

\subsection{Spiked Blood Samples}

Blood samples from non-T. cruzi-infected individuals were mixed with the same volume of GE buffer and processed as clinical samples. A pool of negative samples were spiked with cultured $T$. cruzi trypomastigotes, VD strain. Trypomastigotes were obtained from the supernatants of Vero cell cultures harvested between days 5 and 9 post-infection
Fig. 1 Sample collection diagram during our prospective cohort study. Baseline, at the time of recruitment; End of follow-up, at 9 months of age

\section{Baseline}

(T1)
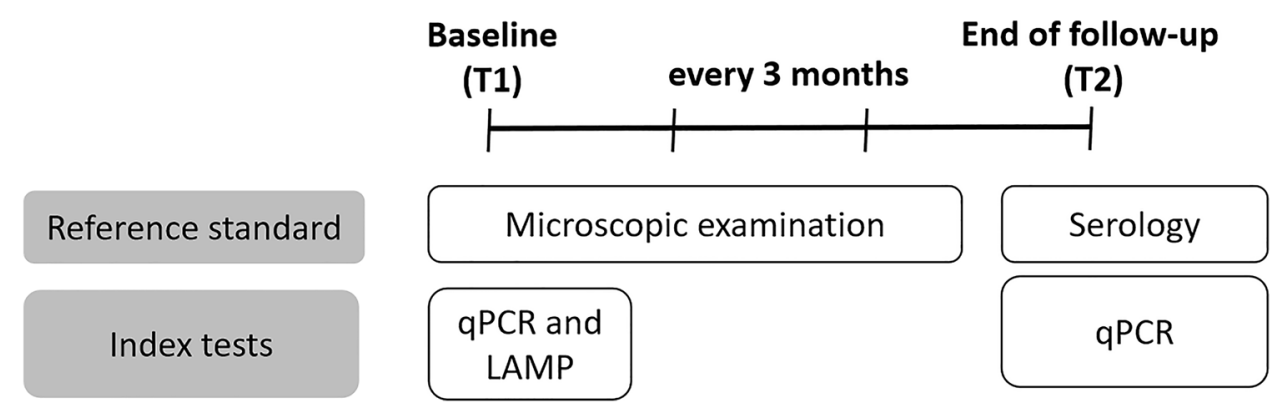
[23]. This strain corresponds to the discrete typing unit (DTU) TcVI and was isolated from a patient with CCD assisted in our service [24]. TcVI was chosen because TcII/V/VI populations have been identified in most patients residing in Argentina [25].

\subsection{Standard Curves}

To build the standard curve for the quantification of parasitic loads, DNA from spiked samples that contained $10^{6}$ trypomastigotes $/ \mathrm{mL}$ was extracted as previously described, followed by serial dilutions to obtain concentrations of $5.10^{5}$ to 0.5 parasite equivalents per milliliter of blood (par. eq. $/ \mathrm{mL}$ ). As diluent, the DNA extracted from a blood sample of a noninfected individual was used. Each dilution was amplified by qPCR in triplicate. To obtain the absolute quantification of parasitic loads from clinical samples, results were interpolated on the standard curve by the StepOne Software v2.3.

\subsection{Quality Assessment of qPCR}

To verify the analytical performance previously reported [22], a replicate test was performed to verify the reported limit of detection (the lowest parasitic load that gives $95 \%$ of positive results). DNA from spiked samples that contained $0.5,2$, and 5 par. eq. $/ \mathrm{mL}$ was extracted and amplified for 10 consecutive days (two replicates for each dilution) in the same manner as reported for the clinical samples.

To ensure the quality of the assay over time, a pool of blood samples from seronegative patients was spiked with cultured trypomastigotes to reach a concentration of 5 par. eq./mL load. Aliquots were stored at $4{ }^{\circ} \mathrm{C}$ to perform a replicate test for each DNA extraction round (positive extraction control). Ct results obtained by qPCR were plotted using quality-control charts.

\subsection{LAMP Reaction}

We have previously validated a LAMP reaction targeted to the $18 \mathrm{~S}$ rRNA genes [14]. Briefly, DNA samples $(5 \mu \mathrm{L})$ were subjected to amplification in reaction mixtures containing 40 pmol of the FIP and BIP primers, 20 pmol of the LF and LB primers, 5 pmol of the $\mathrm{F} 3$ and B3 primers, $1 \mu \mathrm{L}$ (8 units) of Bst DNA polymerase (New England Biolabs, Ipswich, MA, USA), 1X reaction buffer (which is composed of 20 $\mathrm{mM}$ of Tris- $\mathrm{HCl}, 10 \mathrm{mM}$ of $\mathrm{KCl}, 8 \mathrm{mM}$ of $\mathrm{MgSO}_{4}, 10 \mathrm{mM}$ of [NH4] $2 \mathrm{SO}_{4}, 0.1 \%$ Tween-20), $1.4 \mathrm{mM}$ of each dNTP, an additional $8 \mathrm{mM}$ of $\mathrm{MgSO}_{4}$, and $0.5 \mathrm{M}$ of betaine. The reaction was performed at $55{ }^{\circ} \mathrm{C}$ for $60 \mathrm{~min}$, using a heat block $\left(\right.$ Labnet $\left.{ }^{\circledR}\right)$ for amplification. Two negative controls (water and DNA from non-infected human samples) and a positive amplification control (DNA purified from known quantities of $T$. cruzi trypomastigotes) were included in every round. The product was analyzed by visual inspection after the addition of $2 \mu \mathrm{L}$ of the $1000 \times$ concentrated fluorescent dye, SYBR ${ }^{\circledR}$ Green (S7563) [Invitrogen, Eugene, OR, USA]. LAMP results are qualitative and therefore expressed as positive or negative. An immediate change to green fluorescence indicated a positive result, whereas an orange color indicated a negative result.

\subsection{Ethics}

This protocol was reviewed and approved by the Research and Teaching and Bioethics Committees from "Ricardo Gutiérrez" Children's Hospital (CEI No. 10.57). Written informed consent to participate was obtained from each patient's legal representative. All samples were decoded and identified before they were provided for research purposes. Infected patients were treated with benznidazole (100-mg tablets, Abarax ${ }^{\circledR}$; Laboratorio Elea, Buenos Aires, Argentina) at $5-8 \mathrm{mg} / \mathrm{kg}$ body weight, as soon as the diagnosis was confirmed, divided in two daily doses for 60 days [2].

\subsection{Data Analysis}

Results were analyzed using Microsoft Excel and GraphPad Prism software 6.0. Confidence intervals (CIs) were calculated and the $p$ value was considered significant at a value of at least 0.05. Data are expressed as mean \pm standard deviation with a 95\% CI. To evaluate the diagnosis accuracy of the two assays, we calculated sensitivity and specificity, likelihood ratio, and positive and negative predictive values (https://www.medcalc.org/calc/diagnostic_test.php).

\section{Results}

\subsection{Populations and Samples}

A total of 154 infants were referred to our center for CCD diagnosis. Eleven out of the $154(7.1 \%)$ infants were excluded from the analysis because maternal serology was negative or their mother had been previously treated (Fig. 2). Then, 143 (92.9\%) patients were eligible participants to be enrolled in the study. Blood samples for molecular diagnosis from 120 out of these 143 (83.9\%) patients were available for testing. Samples from 23 patients were not available either because no samples were collected ( $n$ $=18$ ) owing to a difficult blood draw or because specimens could not be tested because of inadequate storage with the GE buffer $(n=5)$. All patients were tested by ME at least once. Finally, 102 out of the 120 patients completed the 
follow-up until the $\mathrm{T} 2$ appointment. Thus, the completion rate was $85.0 \%$ (Fig. 2).

Regarding the country of origin of the mothers, $80 / 120$ (66.7\%) were from Bolivia, 26/120 (21.7\%) from Argentina, and 10/120 (8.3\%) from Paraguay. No data of the country of origin were available in 4/120 (3.3\%) women. A total of 12/26 (46.2\%) women from Argentina had been born in Buenos Aires, a non-endemic region for Chagas disease. All infants were born in and remained within the urban limits of Buenos Aires, Argentina, an area free of vector-borne $T$. cruzi transmission without risk of vectorial infection. The mean age of infants at enrollment was 35 days (age range 3-226 days). All patients were asymptomatic, with no cardiac involvement or other Chagas disease-associated pathologies, and no comorbidities or laboratory abnormalities.

At the end of the study, $13 / 120(10.8 \%, 95 \%$ CI 6.4-17.7) infants were diagnosed as CCD infected by the reference standard algorithm (Fig. 2): 9/13 (69.2\%) were detected by ME at baseline (Table 1); 1/13 was diagnosed at a second appointment, during follow-up, before T2 (ID: 101, T1bis; Table 2); and 3/13 (23.1\%) were diagnosed by reactive serology at 9.1, 10.6, and 19.2 months (ID: 061, 053, and 052 respectively; Table 2). The mean age at diagnosis was 4.4 months (standard deviation \pm 5.7 ). A total of 89 patients showed negative serology at the end of the followup. Final serology was performed at a mean age of 11.1 months (standard deviation \pm 4.5 ). For the remaining 18/120 infants, final diagnosis was not available due to loss during follow-up (Fig. 2).

\subsection{Quality-Control Assessment of qPCR}

The qPCR yielded a limit of detection of 2 par. eq. $/ \mathrm{mL}$. For quantitative analysis, the reportable range was $5-5 \times$ $10^{5}$ par. eq. $/ \mathrm{mL}$ and the linear regression analysis showed an efficiency of 0.953 , an $R^{2}$ of 0.999 , and a CV\% (5 par. eq./mL) of 2.7. The quality-control charts allowed monitoring of the quality of the assay over time. Ct values from spiked samples that contained 5 par. eq. $/ \mathrm{mL}$ of blood each round were plotted and Westgard rule analysis discarded the results of only one round during the study.

\subsection{Accuracy of the qPCR and LAMP Assays}

A total of 176 samples were obtained from 120 patients (119 at T1, 55 at T2, and two additional samples [namely T1bis and T2bis] at 3.7 and 19.2 months as a second sample because a negative reference standard but positive qPCR had been obtained). All the 176 samples were tested by qPCR, 17 of which yielded positive results and 159 of which yielded negative results, whereas 121 out of the 176 samples were tested by LAMP, ten of which yielded positive results and 111 of which yielded negative results.
Fig. 2 Flow diagram of infants enrolled in the study. Index tests: qPCR and LAMP; reference standard: microscopic examination before 9 months of age and serology after 9 months. $M D$ molecular diagnosis

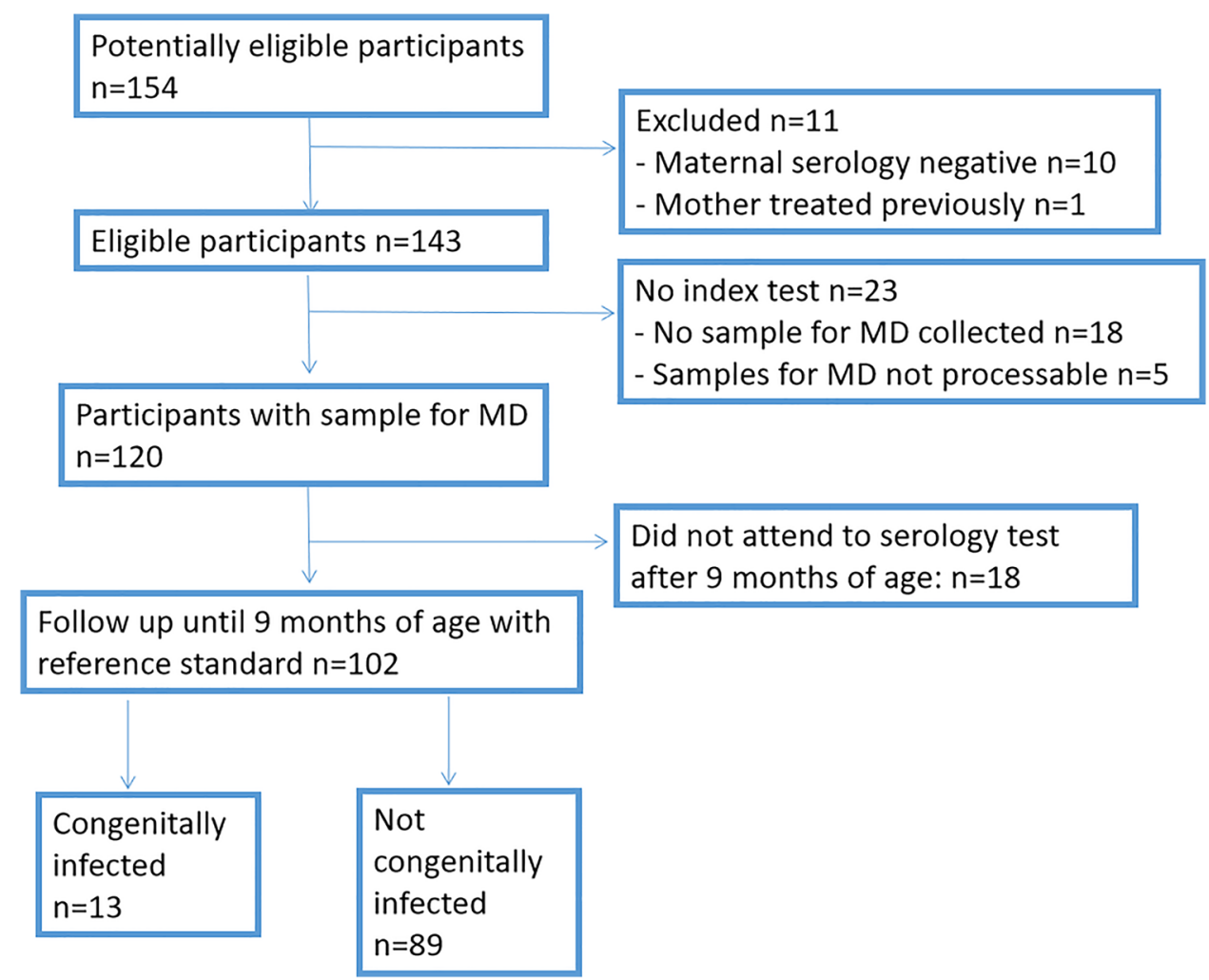


Table 1 Diagnostic accuracy of microscopic examination and molecular tools taking into account the first sample analyzed

\begin{tabular}{lccccll}
\hline Detection method & TP & TN & Sensitivity (95\% CI) & Specificity (95\% CI) & Likelihood ratio (95\% CI) & Predictive value (95\% CI) \\
\hline ME & $9 / 13$ & $89 / 89$ & $69.2 \%(38.6-90.9)$ & $100.0 \%(95.9-100.0)$ & LR + >999 & PPV $=100.0 \%$ \\
& & & & & LR $-0.31(0.14-0.70)$ & NPV $=95.7 \%(90.8-98.1)$ \\
qPCR & $13 / 13^{\mathrm{a}}$ & $89 / 89$ & $100.0 \%(75.3-100.0)$ & $100.0 \%(95.9-100.0)$ & LR $+>999$ & PPV $=100.0 \%$ \\
& & & & & LR -0.00 & NPV $=100.0 \%$ \\
LAMP & $9 / 13^{\mathrm{a}}$ & $89 / 89$ & $69.2 \%(38.6-90.9)$ & $100.0 \%(95.9-100.0)$ & LR $+>999$ & PPV $=100.0 \%$ \\
& & & & & LR $-0.31(0.14-0.70)$ & NPV $=95.7 \%(90.8-98.1)$ \\
\hline
\end{tabular}

$C I$ confidence interval, $L A M P$ isothermal assay, $L R$ - likelihood ratio of a negative test, $L R+$ likelihood ratio of a positive test, $M E$ microscopic examination by microhematocrit method, $N P V$ negative predictive value, $P P V$ positive predictive value, $q P C R$ real-time PCR, $T 1$ baseline, $T 2$ after 9 months of age, $T N$ true negative, $T P$ true positive

${ }^{\mathrm{a}} 12$ samples collected at $\mathrm{T} 1$ and 1 sample collected at $\mathrm{T} 2$

Table 2 Parasitological and serological findings from congenital Chagas disease-infected infants during the follow-up

\begin{tabular}{|c|c|c|c|c|c|c|}
\hline \multirow[t]{2}{*}{ Patient ID } & \multirow[t]{2}{*}{ Appointment } & \multirow[t]{2}{*}{ Age } & \multirow{2}{*}{$\begin{array}{l}\text { Reference standard (ME or } \\
\text { serology) }\end{array}$} & \multicolumn{3}{|c|}{ Index tests } \\
\hline & & & & LAMP & qPCR & $\begin{array}{l}\text { QPCR } \\
\text { (par. eq./ } \\
\mathrm{mL} \text { ) }\end{array}$ \\
\hline 015 & $\mathrm{~T} 1$ & $6 \mathrm{D}$ & Pos & Pos & Pos & 706.2 \\
\hline 032 & $\mathrm{~T} 1$ & $5 \mathrm{D}$ & Pos & Pos & Pos & 23.6 \\
\hline 037 & $\mathrm{~T} 1$ & $9 \mathrm{D}$ & Pos & Pos & Pos & 347.8 \\
\hline 042 & $\mathrm{~T} 1$ & $7.1 \mathrm{M}$ & Pos & Pos & Pos & 4718.3 \\
\hline \multirow[t]{3}{*}{052} & $\mathrm{~T} 1$ & $39 \mathrm{D}$ & Neg & Neg & Pos & NQ \\
\hline & $\mathrm{T} 2$ & $10.5 \mathrm{M}$ & Neg & ND & Pos & 73.6 \\
\hline & $\mathrm{T} 2 \mathrm{bis}$ & $19.2 \mathrm{M}$ & Pos & ND & Pos & 7.8 \\
\hline \multirow[t]{2}{*}{053} & $\mathrm{~T} 1$ & $8 \mathrm{D}$ & $\mathrm{Neg}$ & ND & ND & ND \\
\hline & $\mathrm{T} 2$ & $10.6 \mathrm{M}$ & Pos & Pos & Pos & 2145.3 \\
\hline \multirow[t]{2}{*}{061} & $\mathrm{~T} 1$ & $17 \mathrm{D}$ & Neg & Neg & Pos & NQ \\
\hline & $\mathrm{T} 2$ & $9.1 \mathrm{M}$ & Pos & ND & Pos & 543.1 \\
\hline 084 & $\mathrm{~T} 1$ & $53 \mathrm{D}$ & Pos & Pos & Pos & 257.8 \\
\hline 091 & $\mathrm{~T} 1$ & $4 \mathrm{D}$ & Pos & Pos & Pos & 2058.3 \\
\hline \multirow[t]{2}{*}{101} & $\mathrm{~T} 1$ & $11 \mathrm{D}$ & $\mathrm{Neg}$ & Neg & Pos & 27.5 \\
\hline & T1bis & $3.7 \mathrm{M}$ & Pos & Pos & Pos & 3392.8 \\
\hline 118 & $\mathrm{~T} 1$ & $29 \mathrm{D}$ & Pos & Pos & Pos & 164.7 \\
\hline 140 & $\mathrm{~T} 1$ & $84 \mathrm{D}$ & Pos & Pos & Pos & 1698.0 \\
\hline 141 & $\mathrm{~T} 1$ & $44 \mathrm{D}$ & Pos & Neg & Pos & 320.0 \\
\hline
\end{tabular}

$D$ days, $L A M P$ isothermal assay, $M$ months, $M E$ microscopic examination, $N D$ not done, $N e g$ no detectable parasites or non-reactive serology as appropriate, $N Q$ not quantifiable $(<0.5$ par. eq. $/ \mathrm{mL})$, par. eq. $/ \mathrm{mL}$ parasite equivalents per milliliter of blood, Pos detectable or reactive as appropriate, $q P C R$ real-time PCR, $Q P C R$ quantitative PCR, $T 1$ baseline, $T 2$ after 9 months of age, T1bis and T2bis off-label appointments

${ }^{a}$ Reference standard, ME at baseline (T1 and T1bis) and serology at 9 months old or more if ME was negative (T2 and T2bis)

For the 102 patients who completed the follow-up, and thus the reference standard was available, the diagnosis accuracy of the two molecular tools studied and ME was calculated taking into account the first sample analyzed. Table 1 shows the accuracy assessed as sensitivity, specificity, predictive values, and likelihood ratio.
All 13 infected patients were positive by qPCR in the first sample tested (sensitivity 100\%, 95\% CI 75.3-100.0, Table 1). In one case, the first sample was obtained at $\mathrm{T} 2$ (ID: 053), and the qPCR was also positive. The details of the reference standard and index tests results obtained from the 13 infected patients during the follow-up are 
shown in Table 2. Three infants with negative ME at baseline but CCD diagnosed during the follow-up (see above) were positive at baseline by qPCR, i.e., earlier than with the reference standard (ID: 052, 061, and 101 detected by qPCR at 39, 17, and 11 days old, respectively; Table 2). In infected patients from whom other samples were available during the follow-up, the qPCR remained positive (T1bis, T2, and T2bis, Table 2). However, all 89 non-infected patients were negative by qPCR in both T1 and T2 samples (specificity at baseline $100 \%, 95 \%$ CI 95.9-100.0, Table 1)

LAMP results obtained in this study were detected by naked-eye visualization, as exemplified in Fig. 3. LAMP was positive in $9 / 13$ infected patients in the first sample tested (sensitivity: 69.2\% 95\% CI 38.6-90.9) (Table 1). Eight out of the 13 infected patients yielded positive LAMP results at $\mathrm{T} 1$, whereas four of them yielded negative LAMP results at $\mathrm{T} 1$. In one case, the first sample was obtained at T2 (ID: 053) and yielded a positive result. One of the 13 infected patients was not detectable by LAMP at T1, but was positive at T1bis (ID: 101, Table 2) when parasitic loads increased. All 89 non-infected patients were negative by LAMP (specificity $100.0 \%, 95 \%$ CI 95.9-100.0).

\subsection{Parasitic Loads in Congenitally Infected Patients}

Parasitic loads assessed by quantitative qPCR ranged from not quantifiable (positive but less than 5 par. eq. $/ \mathrm{mL}$ ) to 4718.3 par. eq./mL, using a standard curve from seronegative blood spiked with the VD strain. Parasitic loads exhibited a tendency to increase in infants between 3 and 9 months of age, although there was a very large variation in values (Fig. 4). Moreover, when prior to treatment at least two positive samples were analyzed during the 12 first months of life, the parasitic loads increased (ID: 052, 061, and 101, Table 2). Interestingly, parasites could not be detected by ME or LAMP in T1 samples with non-quantifiable parasitic loads (ID: 052 and 061, Table 2) or in another T1 sample with a low parasitic load (27.4 par. eq./mL, ID:

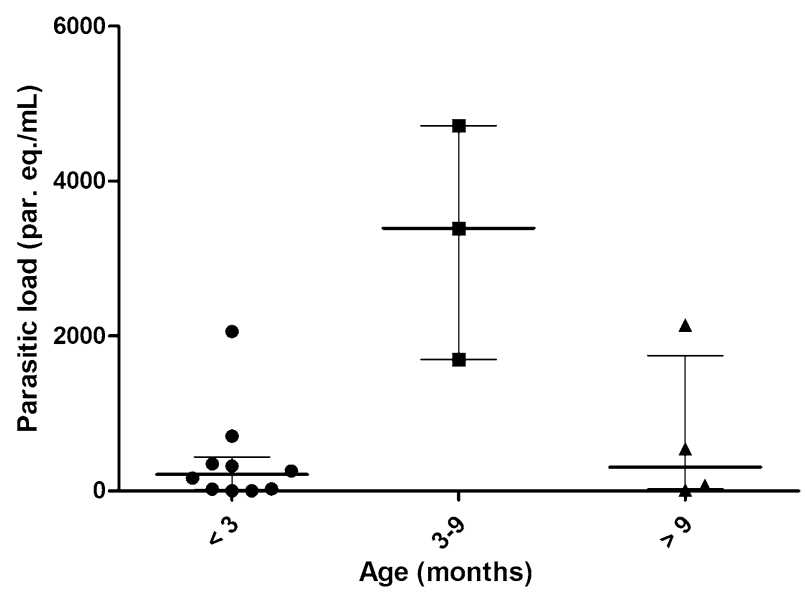

Fig. 4 Comparative analysis of parasitic load in congenital Chagas disease-infected infants on the basis of age, obtained by qPCR assay. Graphs show median and interquartile range. par. eq. $/ \mathrm{mL}$ parasite equivalents per milliliter of blood

101, Table 2). However, the T1 sample from patient ID 032 with 23.6 par. eq. $/ \mathrm{mL}$ showed positive results by both ME and LAMP (Table 2). Additionally, LAMP could not detect T. cruzi DNA in a sample with a parasitic load of 320.0 par. eq./mL (ID: 141, Table 2). However, in one of the patients whose second sample (T1bis) showed an increased parasitic load, both the ME and LAMP turned to positive (ID: 101, Table 2).

\section{Discussion}

In this prospective study, we report the diagnostic accuracy of two molecular tools (a qPCR and a LAMP assay) for the diagnosis of $\mathrm{CCD}$ in a routine diagnosis setting. Although similar studies have been conducted to evaluate the use of qPCR in research settings [26, 27], this is the first work to validate the implementation of a $T$. cruzi LAMP assay for the diagnosis of CCD. Our study evidenced moderate and high overall accuracy of LAMP and qPCR, respectively. The
Fig. 3 Visualization of LAMP results by the naked eye obtained in: tubes 1-3: blood samples obtained from noninfected patients; tube 4: blood sample from an infected patient (ID: 118, Table 2); tube 5: positive amplification control (VD Trypanosoma cruzi stock); tube 6: negative control

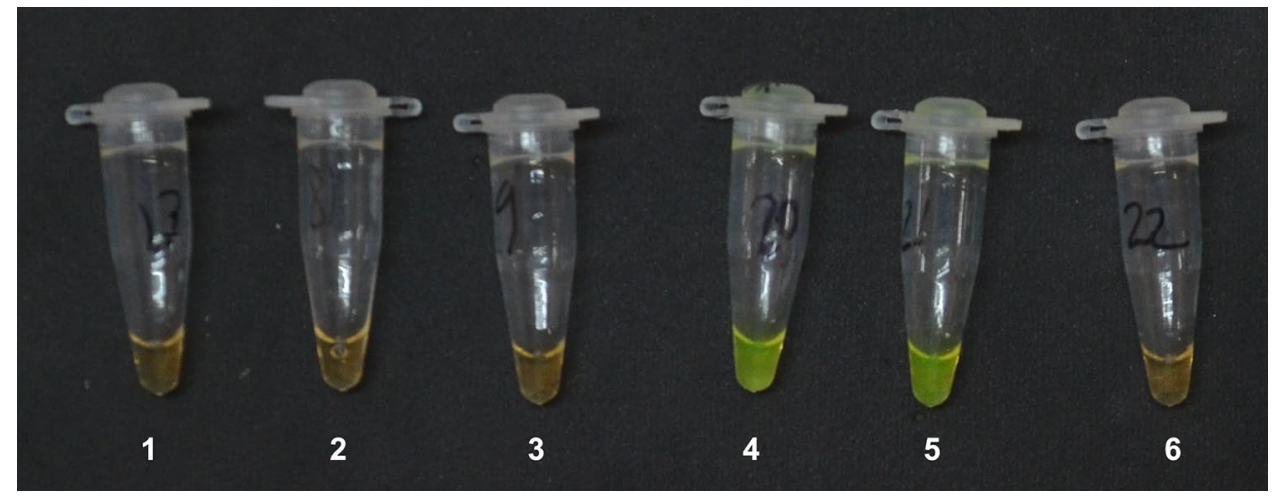


T. cruzi-qPCR proved to be an appropriate tool to anticipate the diagnosis and avoid long follow-up periods.

The transmission rate observed was $10.8 \%$, i.e., higher than that observed in other epidemiological studies [1]. Nevertheless, it should be noted that patients were referred for CCD diagnosis from other centers. This fact may overestimate the transmission rate.

In the metropolitan area of Buenos Aires, most new Chagas disease cases occur by vertical transmission and many infections are currently second-generation congenital cases, meaning that their mothers were themselves congenital cases [28]. In our study, at least 5 out of the 13 infected infants were born to women who had never traveled to endemic areas. In fact, this should be considered by healthcare teams to eliminate the belief that people with Chagas disease are only those migrating from rural or historically endemic areas. Additionally, this reinforces the need to accomplish the guidelines of universal active screening for Chagas disease in pregnant women and their infants as well as to implement the treatment of women of a fertile age to prevent CCD transmission [3, 28, 29].

When pursued in the infants' first months of life, treatment for Chagas disease is safe and effective [2]. The framework for Elimination of Mother-to-Child Transmission of HIV, Syphilis, Hepatitis B, and Chagas (EMTCT plus) aims to increase treatment to $90 \%$ or more infants with CCD [9]. However, using the reference standard algorithm, infants need to be followed up for a prolonged period until the confirmation of a negative or positive serology. In the present study, $15 \%$ of the patients were lost during the follow-up (Fig. 2). This proportion is even higher outside the context of reference centers, reaching 55-80\% in Argentina [5, 8, 10]. Thus, better opportunities for early detection and treatment of CCD should be explored in the context of neonatal monitoring before the mother-newborn pair has left the maternity service (i.e., National Neonatal Screening Program in Argentina). Our data show that qPCR is a potential tool to achieve the goals of EMTCT plus detecting more than $90 \%$ of the infected infants in the first analyzed sample allowing for the implementation of early treatment. Taking into account the evidence from this and another recent study [30], the algorithm for the diagnosis of CCD should be updated to allow qPCR to be used as a tool for early diagnosis when supplies and equipment are available.

Although, since 1998, several studies have shown the high sensitivity of PCR in CCD diagnosis, in most Latin American countries, this tool had not been validated in routine screening. The diverse arguments placed forward to justify the limited use of PCR to diagnose CCD include: limited evidence that molecular tools perform better than the reference standard (parasitology at birth and/or serology 8-12 months after birth), lack of standardization or specificity, complexity, and cost [31]. Our present results showed that both the sensitivity and specificity of qPCR were $100 \%$, with complete agreement with the current diagnostic algorithm (Table 1). The results also showed that, even in the context of a healthcare center, the available qPCR assay has high sensitivity and negative predictive value (NPV), as well as high specificity and positive predictive value (PPV) when used with appropriate quality controls.

The World Health Organization convened Latin American countries to update infant diagnosis practices to ensure early and efficient diagnosis and treatment of CCD [9]. Therefore, early diagnosis of the infection plays an essential role in controlling the disease burden in this population. In this prospective study, qPCR performed in blood samples from infants born to infected mothers was able to detect CCD earlier than LAMP and ME (ID: 052, 061, and 101, Table 2). For a molecular diagnostic tool to be applied in the first days of life, it should be very sensitive because the parasitic loads in some patients are low at baseline and increase in the following appointments (Table 2 and Fig. 4). In fact, there is evidence that blood sampling at 1 month of life improves the sensitivity of diagnosis [27].

Although qPCR is the tool of choice in the most advanced microbiology laboratories, it is not always available in lowresource settings. In a previous analytical study, using this LAMP assay targeted to the 18S rRNA genes, we showed that the use of colorimetric visualization allows an easy judgment of a positive result by the naked eye, allowing a rapid detection of results in seven samples selected from CCD-infected patients with positive ME [14]. This study validates the clinical use of LAMP to detect $T$. cruzi, showing that this assay would be useful in the detection of CCD. LAMP was able to detect infected infants in the first sample tested by colorimetric visualization without requiring complex equipment (sensitivity: $69.2 \%, 95 \%$ CI $38.6-90.9$ and specificity: $100.0 \%$, 95\% CI 95.9-100.0).

However, Besuschio et al. tested a LAMP prototype kit, so far not commercially available, on a series of archival DNA samples from acutely infected patients and found sensitivity and specificity values of $93 \%$ and $100 \%$, respectively $[15,16]$. All the samples they obtained from CCD-infected patients (average age 108 days old) yielded positive LAMP results and the parasitic loads found were between 5.27 and 3063.47 par. eq $/ \mathrm{mL}[15,16]$. Because the performance of the molecular tests will critically depend on the copy number of the target sequences, probably, increased sensitivity was possible because this prototype kit is targeted to satellite DNA sequences (a higher copy number than the $18 \mathrm{~S}$ rRNA genes). To our knowledge, although another LAMP prototype kit exists in Argentina, no results have been published on its performance for the diagnosis of CCD. qPCR and LAMP assays targeting the same sequence would be more appropriate but as these prototype kits are not commercially available, we were limited to evaluating the $18 \mathrm{~S}$ 
rRNA-targeted LAMP that had been previously validated and could be performed as an in-house assay in the laboratory. Future comparative prospective studies must be conducted to determine the clinical validation of these assays.

In this prospective study, LAMP was found to perform similarly to ME. Only one sample, with a moderate parasitic load, was positive by ME but negative by LAMP (ID: 141, 320 eq. par./mL, Table 2). Although T. cruzi DTUs in clinical samples were not analyzed, our previous analytical study using this LAMP assay was carried out with purified DNA from reference $T$. cruzi strains belonging to the different DTUs and showed no significant differences in the analytical sensitivities among them [14]. Technical problems such as DNA degradation during storage might have been a possible cause for this false-negative LAMP finding. Furthermore, because the LAMP assay available for this study did not have IPC and we did not have fluorescence reading equipment, a limitation of this study is that some low-level LAMP amplification may have been missed by the naked eye reading or by a reaction inhibition.

In this study, qPCR was more sensitive than LAMP. Nevertheless, although not ideal, this is a simple and practical molecular tool that could be performed in healthcare facilities without expensive equipment or trained microscopists, providing results rapidly through detection by the naked eye [31].

In our work, the quality of the qPCR and LAMP assays was checked and evaluated continuously by an in-house quality-control process. This allowed us to rule out invalid results. In contrast to microscopy, molecular diagnosis allows the monitoring of results by a program of quality control following the EMTCT plus guidelines to ensure high-quality testing [9].

Molecular diagnostic tools such as those evaluated in the present study have numerous advantages, including increased sensitivity and specificity and a simpler standardization of diagnostic procedures. DNA samples can also be stored and used for genetic characterization and molecular typing, providing a valuable tool for surveys and surveillance studies. The COVID-19 pandemic has proven the worth of rapid implementation of molecular diagnostic tools. All of these installed capabilities of molecular laboratories in the health system should be profited, in the near future, for diagnosis of neglected diseases such as Chagas disease.

The study has potential limitations. As Chagas disease has a low rate of congenital transmission, the number of infected children is small, according to the CIs of the LAMP, in nonoptimal conditions, the sensitivity could be $38.6 \%$, the same as an ME (Table 1). In addition, the infants included had been referred to the reference center, the youngest being 3 days old. A recent study in neonates has shown lower sensitivity in neonates studied on the first day of life [30]. These limitations could be overcome in multicenter studies that allow an evaluation of diagnostic accuracy including neonatology services, different operators in the reading of the $\mathrm{ME}$, and a greater number of samples.

\section{Conclusions}

To the best of our knowledge, this is the first study jointly reporting the sensitivity, specificity, PPV, and NPV of LAMP and qPCR in the diagnosis of CCD. The NPV identified the limitations of negative LAMP results for ruling out infection in non-infected infants; however, high specificity and PPV were obtained. As in other studies, the high sensitivity, specificity, PPV, and NPV values highlighted qPCR as a valuable tool in the earlier diagnosis of CCD.

Acknowledgements We thank Dr. Alejandro Schijman (INGEBICONICET) for the linearized p-Zero plasmid containing a sequence of Arabidopsis thaliana used as IPC. We are also grateful to Dr. Ernesto Gulin for culturing T. cruzi parasites necessary for constructing standard curves and positive controls.

\section{Declarations}

Funding Agencia Nacional de Promoción Científica y Tecnológica, Argentina (FONARSEC FITS-Chagas 3-2012), ANLIS-Malbrán (FOCANLIS NRU: 1106, 2013, and NRU: 1524, 2015). MMCB, GM, AMR and JA are members of the Research Career of CONICET (Consejo Nacional de Investigaciones Científicas y Técnicas). The funders had no role in the study design, data collection and analysis, decision to publish, or preparation of the manuscript.

Conflicts of Interest/Competing Interests The authors have no competing interests to declare.

Ethics Approval This protocol was reviewed and approved by the Research \& Teaching and Bioethics Committees from "Ricardo Gutiérrez" Children's Hospital (CEI No. 10.57).

Consent to Participate Written informed consent to participate was obtained from each patient's legal representative.

Consent for Publication Patient's legal representative signed informed consent regarding publishing their data.

Availability of Data and Material The data that support the findings and conclusions of this study are available on request from the corresponding author.

Code Availability Not applicable.

Authors' Contributions MMCB and RR planned the study and experiments, analyzed the data, and performed the molecular assays. MMCB and CK planned and performed the qPCR experiments and analysis, MMCB, RR, and AMR planned and performed the LAMP experiments and analysis. GB and ID performed and analyzed the serological and ME procedures. NG, SM, and GM collected samples and performed the clinical follow-up of the infants. MMCB and RR wrote the first draft of the manuscript. JA planned the clinical study and read and approved the manuscript. All authors read and approved the final manuscript. 


\section{References}

1. Carlier Y, Altcheh J, Angheben A, Freilij H, Luquetti AO, Schijman AG, et al. Congenital Chagas disease: updated recommendations for prevention, diagnosis, treatment, and follow-up of newborns and siblings, girls, women of childbearing age, and pregnant women. PLoS Negl Trop Dis. 2019. https://doi.org/10.1371/journ al.pntd.0007694

2. Altcheh J, Moscatelli G, Mastrantonio G, Moroni S, Giglio N, Marson ME, et al. Population pharmacokinetic study of benznidazole in pediatric Chagas disease suggests efficacy despite lower plasma concentrations than in adults. PLoS Negl Trop Dis. 2014. https://doi.org/10.1371/journal.pntd.0002907.

3. Moscatelli G, Moroni S, Bournissen FG, González N, Ballering G, Schijman A, et al. Longitudinal follow up of serological response in children treated for Chagas disease. PLoS Negl Trop Dis. 2019. https://doi.org/10.1371/journal.pntd.0007668.

4. Freilij H, Altcheh J. Congenital Chagas' disease: diagnostic and clinical aspects. Clin Infect Dis. 1995;21(3):551-5.

5. De Rissio AM, Riarte AR, García MM, Esteva MI, Quaglino M, Ruiz AM. Congenital Trypanosoma cruzi infection: efficacy of its monitoring in an urban reference healthcare center in a nonendemic area of Argentina. Am J Trop Med Hyg. 2010. https:// doi.org/10.4269/ajtmh.2010.08-0383.

6. Carlier Y, Truyens C. Congenital Chagas disease as an ecological model of interactions between Trypanosoma cruzi parasites, pregnant women, placenta and fetuses. Acta Trop. 2015. https:// doi.org/10.1016/j.actatropica.2015.07.016.

7. Carlier Y, Torrico F, Sosa-Estani S, Russomando G, Luquetti A, Freilij H, et al. Congenital Chagas disease: recommendations for diagnosis, treatment and control of newborns, siblings and pregnant women. PLoS Negl Trop Dis. 2011. https://doi.org/10. 1371/journal.pntd.0001250.

8. Danesi E, Fabbro DL, Segura EL, Sosa-Estani S. Higher congenital transmission rate of Trypanosoma cruzi associated with family history of congenital transmission. Rev Soc Bras Med Trop. 2020. https://doi.org/10.1590/0037-8682-0560-2019.

9. Organization PAH. EMTCT plus: framework for elimination of mother-to-child transmission of HIV, syphilis, hepatitis B, and Chagas. Washington: PAHO; 2017.

10. Velázquez EB, Rivero R, De Rissio AM, Malagrino N, Esteva MI, Riarte AR, et al. Predictive role of polymerase chain reaction in the early diagnosis of congenital Trypanosoma cruzi infection. Acta Trop. 2014;137:195-200. https://doi.org/10. 1016/j.actatropica.2014.05.016.

11. Russomando G, de Tomassone MM, de Guillen I, Acosta N, Vera N, Almiron M, et al. Treatment of congenital Chagas' disease diagnosed and followed up by the polymerase chain reaction. Am J Trop Med Hyg. 1998. https://doi.org/10.4269/ajtmh. 1998.59.487.

12. Schijman AG, Altcheh J, Burgos JM, Biancardi M, Bisio M, Levin MJ, et al. An etiological treatment of congenital Chagas' disease diagnosed and monitored by the polymerase chain reaction. J Antimicrob Chemother. 2003;52:441-9.

13. Bern C, Verastegui M, Gilman RH, Lafuente C, Galdos-Cardenas G, Calderon M, et al. Congenital Trypanosoma cruzi transmission in Santa Cruz, Bolivia. Clin Infect Dis. 2009. https:// doi.org/10.1086/648070.

14. Rivero R, Bisio M, Velázquez EB, Esteva MI, Scollo K, González NL, et al. Rapid detection of Trypanosoma cruzi by colorimetric loop-mediated isothermal amplification (LAMP): a potential novel tool for the detection of congenital Chagas infection. Diagn Microbiol Infect Dis. 2017;89(1):2014-6. https:// doi.org/10.1016/j.diagmicrobio.2017.06.012.
15. Besuschio SA, Murcia ML, Benatar AF, Monnerat S, Cruz I, Picado A, et al. Analytical sensitivity and specificity of a loopmediated isothermal amplification (LAMP) kit prototype for detection of Trypanosoma cruzi DNA in human blood samples. PLoS Negl Trop Dis. 2017;11(7):e0005779. https://doi.org/10. 1371/journal.pntd.0005779.

16. Besuschio SA, Picado A, Muñoz-Calderón A, Wehrendt DP, Fernández M, Benatar A, et al. Trypanosoma cruzi loop-mediated isothermal amplification (Trypanosoma cruzi loopamp) kit for detection of congenital, acute and Chagas disease reactivation. PLoS Negl Trop Dis. 2020;14(8):1-17. https://doi.org/10.1371/ journal.pntd.0008402.

17. Duffy T, Cura CI, Ramirez JC, Abate T, Cayo NM, Parrado $\mathrm{R}$, et al. Analytical performance of a multiplex real-time PCR assay using TaqMan probes for quantification of Trypanosoma cruzi satellite DNA in blood samples. PLoS Negl Trop Dis. 2013;7(1):e2000. https://doi.org/10.1371/journal.pntd.0002000.

18. Cohen JF, Korevaar DA, Altman DG, Bruns DE, Gatsonis CA, Hooft L, STARD, et al. Guidelines for reporting diagnostic accuracy studies: explanation and elaboration. BMJ Open. 2015;6:e012799. https://doi.org/10.1136/bmjopen-2016-012799.

19. Ministerio de Salud de la Nación. Enfermedad de Chagas. Guía para la atención al paciente infectado con Trypanosoma cruzi. CABA; 2018.

20. Freilij H, Muller L, Cappa SMG. Direct micromethod for diagnosis of acute and congenital Chagas' disease. J Clin Microbiol. 1983;18(2):327-30.

21. Organización Panamericana de la Salud. Guía para el diagnóstico y el tratamiento de la enfermedad de Chagas. Washington, DC: OPS. 2018. https://iris.paho.org/handle/10665.2/4965. Accessed 5 Aug 2021.

22. Ramirez JC, Cura CI, da Cruz MO, Lages-Silva E, Juiz N, Pavia P, et al. Analytical validation of quantitative real-time PCR methods for quantification of Trypanosoma cruzi DNA in blood samples from Chagas disease patients. J Mol Diagn. 2015;17(5):605-15. https://doi.org/10.1016/j.jmoldx.2015.04.010.

23. Brossas JY, Gulin JEN, Bisio MMC, Chapelle M, Marinach-Patrice C, Bordessoules M, et al. Secretome analysis of Trypanosoma cruzi by proteomics studies. PLoS ONE. 2017;12(10):e0185504. https://doi.org/10.1371/journal.pone. 0185504.

24. Gulin JEN, Bisio M, Rocco DM, Altcheh J, Solana ME, Bournissen FG. Molecular and biological characterization of a highly pathogenic Trypanosoma cruzi strain isolated from a patient with congenital infection. Exp Parasitol. 2018;186:50-8. https://doi. org/10.1016/j.exppara.2018.02.002.

25. Cura CI, Duffy T, Lucero RH, Bisio M, Péneau J, Jimenez-Coello $\mathrm{M}$, et al. Multiplex real-time PCR assay using TaqMan probes for the identification of Trypanosoma cruzi DTUs in biological and clinical samples. PLoS Negl Trop Dis. 2015;9(5):e0003765. https://doi.org/10.1371/journal.pntd.0003765.

26. Bua J, Volta BJ, Perrone AE, Scollo K, Velázquez EB, Ruiz AM, et al. How to improve the early diagnosis of Trypanosoma cruzi infection: relationship between validated conventional diagnosis and quantitative DNA amplification in congenitally infected children. PLoS Negl Trop Dis. 2013;7:e2476. https://doi.org/10.1371/ journal.pntd.0002476.

27. Messenger LA, Gilman RH, Verastegui M, Galdos-Cardenas G, Sanchez G, Valencia E, Working Group on Chagas Disease in Bolivia and Peru, et al. Toward improving early diagnosis of congenital Chagas disease in an endemic setting. Clin Infect Dis. 2017;65(2):268-75. https://doi.org/10.1093/cid/cix277.

28. Moscatelli G, Moroni S, Bournissen FG, Ballering G, Bisio M, Freilij $\mathrm{H}$, et al. Prevention of congenital Chagas through treatment 
of girls and women of childbearing age. Mem Inst Oswaldo Cruz. 2015;110(4):507-9. https://doi.org/10.1590/0074-02760140347.

29. Lascano F, Bournissen FG, Altcheh J. Review of pharmacological options for the treatment of Chagas disease. Br J Clin Pharmacol. 2020. https://doi.org/10.1111/bcp.14700.

30. Benatar AF, Danesi E, Besuschio SA, Bortolotti S, Cafferata ML, Ramirez JC, Congenital Chagas Disease Study Group, et al. Prospective multicenter evaluation of real time PCR kit prototype for early diagnosis of congenital Chagas disease. EBioMedicine. 2021;69:103450. https://doi.org/10.1016/j.ebiom.2021.103450.

31. Picado A, Cruz I, Redard-Jacot M, Schijman AG, Torrico F, Sosa-Estani S, et al. The burden of congenital Chagas disease and implementation of molecular diagnostic tools in Latin America. BMJ Glob Health. 2018;3(5):e001069. https://doi.org/10.1136/ bmjgh-2018-001069. 